\title{
In vitro activity of antimicrobial combinations against multidrug-resistant Pseudomonas aeruginosa
}

\author{
Denissani Aparecida Ferrari dos Santos Lima ${ }^{[1]}$, \\ Margarida Maria Passeri do Nascimento ${ }^{[1]}$, Lúcia Helena Vitali ${ }^{[1]}$ \\ and Roberto Martinez ${ }^{[1]}$
}

[1]. Departamento de Clínica Médica, Faculdade de Medicina de Ribeirão Preto, Universidade de São Paulo, São Paulo, SP.

\begin{abstract}
Introduction: Pseudomonas aeruginosa isolates related to nosocomial infections are often resistant to multiple antibacterial agents. In this study, antimicrobial combinations were evaluated to detect in vitro synergy against clinical isolates of $P$. aeruginosa. Methods: Four clinical P. aeruginosa isolates were selected at random among other isolates from inpatients treated at the public University hospital in Ribeirão Preto, SP, Brazil. Two isolates were susceptible to imipenem (IPM-S) and several other antimicrobials, while the other two isolates were imipenem and multidrug resistant (IPM-R). The checkerboard method was used to assess the interactions between antimicrobials. Results: Combinations of imipenem or other anti-Pseudomonas drugs with complementary antibiotics, such as aminoglycosides, fosfomycin and rifampin, reached synergy rates of $20.8 \%, 50 \%, 62.5 \%$ and $50 \%$ for the two IPM-S and two IPM-R Pseudomonas isolates, respectively. Imipenem, piperacillin-tazobactam and ceftazidime yielded a greater synergy rate than cefepime or ciprofloxacin. Synergist combinations were more commonly observed when the complementary drug was tobramycin (65\%) or fosfomycin (57\%). Conclusions: Some antibacterial combinations led to significant reductions of the minimum inhibitory concentrations of both drugs, suggesting that they could be clinically applied to control infections caused by multidrug-resistant $P$. aeruginosa.
\end{abstract}

Keywords: Pseudomonas aeruginosa. Antimicrobial synergy. Beta-lactam agents. Fosfomycin. Tobramycin. Rifampin.

\section{INTRODUCTION}

A universal tendency to bacterial resistance to antimicrobials has been observed since the beginning of antibiotic therapy. Pseudomonas aeruginosa is a microorganism that is particularly difficult to control because it causes opportunistic and nosocomial infections, it is non-susceptible to several antimicrobials and it develops progressive resistance to new drugs $^{1}$. Over the last few years, carbapenemic drugs have become important therapeutic resources for the control of $P$. aeruginosa infections. However, growing resistance to imipenem and other carbapenems has been observed, and multidrug-resistance has become more common $^{2,3}$.

A therapeutic strategy against $P$. aeruginosa is the use of antimicrobial combinations to delay the selection of resistant bacterial clones and to obtain a synergistic antibacterial action ${ }^{4}$. The combination of beta-lactam antibiotics and aminoglycosides has been recommended for the treatment of infected patients.

Address to: Dr. Roberto Martinez. FMRP/USP. Av Bandeirantes 3900, 14048-900 Ribeirão Preto, SP, Brasil.

Phone: 5516 3602-2468

e-mail: rmartine@fmrp.usp.br

Received 23 January 2013

Accepted 15 May 2013
Antimicrobials are also combined as a method to recover the efficacy of drugs to which $P$. aeruginosa has become resistant. When the interaction is synergistic, reductions of the minimum inhibitory concentrations (MICs) of both antimicrobials occur, eventually rendering the microorganism susceptible to the levels of antimicrobials found in the blood and tissues ${ }^{5}$.

This investigation was motivated by the high frequency of nosocomial infections caused by multidrug-resistant P. aeruginosa in a Brazilian emergency and trauma care hospital. The objective was to evaluate the in vitro susceptibility of $P$. aeruginosa by testing antimicrobials that are known to combat $P$. aeruginosa, combined with other potentially active drugs, particularly fosfomycin and rifampin.

\section{METHODS}

Pseudomonas aeruginosa was isolated from the blood $(\mathrm{n}=3)$ and urine $(\mathrm{n}=1)$ of patients who were admitted to the Emergency Unit of the University Hospital, Faculty of Medicine of Ribeirão Preto, University of São Paulo, Ribeirão Preto, SP, Brazil. The isolates were selected at random among other clinical isolates and were identified by the automated microbiology system Vitek (Biomérieux, Jacarepaguá, Brazil). Two isolates ( $8 \mathrm{~S}$ and $34 \mathrm{~S}$ ) were susceptible to imipenem and to several other antimicrobials, while the other two isolates (46R and 72R) were imipenem and multidrug resistant. The four strains were subcultured in brain heart infusion broth (Oxoid, Hampshire, 
England), plated on Müeller Hinton agar (Oxoid, Hampshire, England ) and then stored at $-70^{\circ} \mathrm{C}$ in soy trypticase broth with $15 \%$ glycerol. Aliquots for the tests were removed from this stock, and bacterial growth was recovered by subculture in brain heart infusion broth for 24 hours.

\section{Antimicrobial drugs}

Imipenem, gentamicin, fosfomycin and polymyxin B powder were obtained from Sigma, USA(St. Louis, MO, USA) and were diluted in sterile water for the preparation of the stock solutions. Stock solutions of other drugs were prepared by dilution in sterilized water from the following commercially available medications: ceftazidime (Kefadin, ABL, São Paulo, Brazil), piperacillin-tazobactam (Taz-Pen, Cellofarm, Brazil), cefepime (ABL, São Paulo, Brazil), tobramycin (Tobramina, ABL, São Paulo, Brazil), ciprofloxacin (Ciprobacter/Isofarma, Ceará, Brazil) and rifampin (Rifaldin/Sanofi-Aventis, Paris, France). The stock solutions were stored at $-70^{\circ} \mathrm{C}$, except for imipenem, which was prepared immediately before use. The various drug concentrations were obtained by successive two-fold dilutions in sterile water. The antimicrobial solutions were validated by determination of the respective MICs against P. aeruginosa ATCC 27853.

\section{Broth microdilution susceptibility test}

The method standardized by the Clinical and Laboratory Standards Institute (CLSI) ${ }^{6}$ was used to determine the MICs of the antimicrobials for the selected clinical isolates and for P. aeruginosa ATCC 27853.

Briefly, Müeller Hinton broth that was adjusted for cations (Oxoid, Hampshire, England) was added to the wells of microtiter plates (TPP, Zellkultur, Trasadingen, Switzerland). Solutions with serial dilutions of antimicrobial concentrations were added at variable ranges according to the drug and isolate tested. Finally, $P$. aeruginosa from a 24-hour subculture in Müeller Hinton broth (Oxoid) was adjusted for a turbidity equivalent to 0.5 on the McFarland scale and then diluted to obtain a final inoculum of $2 \times 10^{5}$ colony-forming units (CFU)/ $\mathrm{mL}$. The plates were covered with plastic film, and after 24 hours of incubation at $35^{\circ} \mathrm{C}$, the absorbance of each well of the microtiter plate was measured with a microplate reader that was adjusted for a wavelength of $490 \mathrm{~nm}$.

The criterion used for MIC determination was the lowest antimicrobial concentration with absorbance corresponding to $\leq$ $20 \%$ of the mean absorbance of the control bacterial growth wells (without the addition of the antimicrobial). CLSI-determined breakpoints ${ }^{7}$ were used to evaluate the bacterial susceptibility to antimicrobials, with the exception of fosfomycin. Susceptibility to this drug was analyzed according to the European Committee on Antimicrobial Susceptibility Testing (EUCAST) criterion for Enterobacteriaceae ${ }^{8}$.

\section{Susceptibility test to combined drugs}

The checkerboard method ${ }^{9}$ was used to assess the eventual synergy between antimicrobials against the two imipenemsusceptible and the two multidrug-resistant $P$. aeruginosa isolates. Each drug combination was evaluated in duplicate with each bacterial isolate. The MIC of each separate drug was determined on different sides of the same plate. Wells were used for positive controls (only bacterial inoculum and Müeller Hinton broth) and for negative controls (only combined and separate antimicrobials and Müeller Hinton broth). The combined drugs were tested at different concentrations inside the microplate. A Pseudomonas aeruginosa subculture in Müller Hinton broth was adjusted to obtain a final inoculum of $2 \times 10^{5}$ $\mathrm{CFU} / \mathrm{mL}$ in the well. The plates were covered and incubated at $35^{\circ} \mathrm{C}$ for 24 hours, and the absorbance of each well was then measured with a microplate reader adjusted for a wavelength of $490 \mathrm{~nm}$. The MIC of the separate or combined antimicrobials was defined as the lowest concentration of the drugs with an absorbance that was $\leq 20 \%$ of the mean absorbance of the bacterial growth in the control wells.

\section{Analysis of antimicrobial interaction}

Interaction was analyzed separately for each antimicrobial combination (drugs A and B) and bacterial isolate. The fractional inhibitory concentration of drug A (FIC[A]) was calculated as the following ratio: MIC obtained with drug A combined with the second drug/MIC of drug A alone. FIC[B] was obtained using a similar calculation. The FIC(A) + FIC(B) sum resulted in the FIC index (FICI), representative of the interaction of the two antimicrobials. The following criteria were adopted when the MIC value exceeded the standardized range of drug concentrations: 1) for an MIC above the range limit, the FIC was calculated with the next highest drug concentration, estimated by successive two-fold dilutions; and 2) when the MIC was equal to or below the lowest drug concentration evaluated, this value was used to calculate the FIC. The following criteria of interpretation were adopted: 1) FICI $\leq 0.5$ indicates synergy between the two antimicrobials; 2) FICI $>0.5$ and $\leq 4.0$ indicates indifference; and 3) FICI $>4$ indicates antagonism 9 . The lowest FICI value obtained in the checkerboard test was considered to be representative of the interaction of the two antimicrobials against the respective $P$. aeruginosa isolate, regardless of the concentrations of the two drugs.

\section{Statistical analysis}

The chi-square test was used to analyze the differences in the synergy rates obtained between the bacterial strains and the antimicrobials tested, with the level of significance set at 0.05 .

\section{RESULTS}

The two imipenem-susceptible strains ( $8 \mathrm{~S}$ and $34 \mathrm{~S}$ ) exhibited susceptibility to other anti-Pseudomonas drugs but were resistant to fosfomycin and rifampin. The two imipenemresistant isolates (46R and $72 \mathrm{R}$ ) were also resistant to all antimicrobials evaluated (the MIC of the 46R strain was near the breakpoint with respect to ceftazidime and piperacillintazobactam). The four P. aeruginosa isolates were all susceptible to polymyxin B ( $\mathrm{MIC}=1$ to $2 \mu \mathrm{g} / \mathrm{mL}$ ).

The FICI for the various drug combinations indicated synergic or indifferent activity, with no antagonism being 
TABLE 1 - In vitro synergic combinations between pairs of antimicrobials against two imipenem-susceptible Pseudomonas aeruginosa-minimum inhibitory concentration (MIC) of the isolate or combined drugs and fractional inhibitory concentration index (FICI).

\begin{tabular}{|c|c|c|c|c|}
\hline \multirow[b]{3}{*}{ Strain } & \multicolumn{4}{|c|}{$\operatorname{MIC}(\mu \mathrm{g} / \mathrm{mL})$} \\
\hline & drugs & alone & combined & \\
\hline & $(\mathrm{A} / \mathrm{B})$ & $(\mathrm{A} / \mathrm{B})$ & $(\mathrm{A} / \mathrm{B})$ & FICI \\
\hline $8 \mathrm{~S}$ & IPM/TOB & $2 / 0.5$ & $0.5 / 0.13$ & 0.500 \\
\hline $8 \mathrm{~S}$ & IPM/FOSF & $1 / 512$ & $\leq 0.13 / 16$ & 0.161 \\
\hline $8 \mathrm{~S}$ & IPM/RIF & $2 / 32$ & $0.5 / \leq 4$ & 0.375 \\
\hline $8 \mathrm{~S}$ & PIP-T/TOB & $4 / 0.5$ & $0.5 / \leq 0.13$ & 0.375 \\
\hline $8 \mathrm{~S}$ & PIP-T/FOSF & $4 / 512$ & $1 / 16$ & 0.281 \\
\hline $34 \mathrm{~S}$ & IPM/TOB & $2 / 1$ & $0.5 / 0.25$ & 0.500 \\
\hline $34 \mathrm{~S}$ & IPM/FOSF & $1 / 512$ & $0.25 / 64$ & 0.375 \\
\hline $34 \mathrm{~S}$ & IPM/RIF & $2 / 32$ & $0.5 / 8$ & 0.500 \\
\hline $34 \mathrm{~S}$ & PIP-T/GEN & $16 / 2$ & $2 / \leq 0.5$ & 0.375 \\
\hline $34 \mathrm{~S}$ & PIP-T/TOB & $16 / 1$ & $\leq 0.13 / \leq 0.06$ & 0.068 \\
\hline $34 \mathrm{~S}$ & PIP-T/FOSF & $8 / 512$ & $\leq 0.13 / 64$ & 0.141 \\
\hline $34 \mathrm{~S}$ & CEFTA/GEN & $4 / 2$ & $0.25 / 0.5$ & 0.313 \\
\hline $34 \mathrm{~S}$ & CEFTA/TOB & $4 / 2$ & $1 / \leq 0.5$ & 0.500 \\
\hline $34 \mathrm{~S}$ & CEFTA/FOSF & $8 />512$ & $1 / 32$ & 0.156 \\
\hline $34 \mathrm{~S}$ & CEFTA/RIF & $8 / 64$ & $1 / \leq 8$ & 0.250 \\
\hline $34 \mathrm{~S}$ & $\mathrm{CIP} / \mathrm{FOSF}$ & $0.25 />512$ & $0.06 / 16$ & 0.256 \\
\hline $34 \mathrm{~S}$ & TOB/RIF & $2 / 64$ & $0.5 / 16$ & 0.500 \\
\hline \multicolumn{5}{|c|}{$\begin{array}{l}\text { IPM: imipenem; PIP-T: piperacillin-tazobactam; CEFTA: ceftazidime; } \\
\text { TOB: tobramycin; GEN: gentamicin; CIP: ciprofloxacin; FOSF: fosfomycin; } \\
\text { RIF: rifampin. }\end{array}$} \\
\hline \multicolumn{5}{|c|}{$\begin{array}{l}\text { observed. The MIC value of an antimicrobial generally } \\
\text { decreased when a second drug was present in subinhibitory } \\
\text { concentrations, but in general, the MIC reductions were } \\
\text { discrete. For the synergic antimicrobial combinations, the } \\
\text { initial and the lowest MIC values obtained in the presence of } \\
\text { another drug acting jointly are listed in Table } 1 \text { (imipenem- } \\
\text { susceptible isolates) and Table } 2 \text { (imipenem-resistant } \\
\text { isolates). Some drug combinations led to a great MIC } \\
\text { reduction, such that the new MIC of both drugs was reduced } \\
\text { below the respective breakpoint. Table } 3 \text { shows that there } \\
\text { was synergy in } 46 \% \text { of the combinations of all drugs for the } \\
\text { four isolates tested. Tobramycin }(65 \%) \text { and fosfomycin }(57 \%) \\
\text { were more effective as the second drug of the combinations } \\
\text { than gentamicin ( } 25 \%) \text { and rifampin ( } 36 \%) \text { in terms of } \\
\text { synergistic action against } P \text {. aeruginosa. Synergy with } \\
\text { these drugs was obtained more frequently when imipenem, } \\
\text { piperacillin-tazobactam and ceftazidime were used as the } \\
\text { first anti-Pseudomonas drugs (p }<0.05) \text {. The rate of synergy } \\
\text { obtained with all antimicrobial combinations for isolates } 8 \mathrm{~S} \text {, } \\
34 \mathrm{~S}, 46 \mathrm{R} \text { and } 72 \mathrm{R} \text { reached } 20.8 \%(5 / 24), 50 \%(12 / 24), 62.5 \% \\
(15 / 24) \text { and } 50 \%(12 / 24) \text {, respectively (p }<0.05) \text {. }\end{array}$} \\
\hline
\end{tabular}

TABLE 2 - In vitro synergic combinations between pairs of antimicrobials against two imipenem-resistant $P$ seudomonas aeruginosa-minimum inhibitory concentration (MIC) of the isolate or combined drugs and fractional inhibitory concentration index (FICI).

\begin{tabular}{|c|c|c|c|c|}
\hline \multirow[b]{2}{*}{ Strain } & \multicolumn{4}{|c|}{$\mathrm{MIC}(\mu \mathrm{g} / \mathrm{mL})$} \\
\hline & $\begin{array}{l}\text { dugs } \\
(\mathrm{A} / \mathrm{B}) \\
\end{array}$ & $\begin{array}{l}\text { alone } \\
(\mathrm{A} / \mathrm{B}) \\
\end{array}$ & $\begin{array}{c}\text { combined } \\
(\mathrm{A} / \mathrm{B}) \\
\end{array}$ & FICI \\
\hline $46 \mathrm{R}$ & IPM/TOB & $32 />256$ & $2 / 64$ & 0.188 \\
\hline $46 \mathrm{R}$ & IMP/FOSF & $32 / 256$ & $4 / 32$ & 0.250 \\
\hline $46 \mathrm{R}$ & PIP-T/GEN & $64 />512$ & $16 / 256$ & 0.500 \\
\hline $46 \mathrm{R}$ & PIP-T/TOB & $128 / 256$ & $4 / \leq 8$ & 0.063 \\
\hline $46 \mathrm{R}$ & PIP-T/FOSF & $128 / 256$ & $16 / \leq 8$ & 0.156 \\
\hline $46 \mathrm{R}$ & PIP-T/RIF & $64 / 16$ & $16 / \leq 4$ & 0.500 \\
\hline $46 \mathrm{R}$ & CEFTA/TOB & $8 / 256$ & $2 / 32$ & 0.375 \\
\hline $46 \mathrm{R}$ & CEFTA/FOSF & $16 / 512$ & $4 / 64$ & 0.375 \\
\hline $46 \mathrm{R}$ & CEFTA/RIF & $16 / 32$ & $4 / \leq 8$ & 0.500 \\
\hline $46 \mathrm{R}$ & CPM/GEN & $>512 / 512$ & $32 / \leq 8$ & 0.047 \\
\hline $46 \mathrm{R}$ & CPM/TOB & $>512 / 256$ & $\leq 1 / \leq 8$ & 0.033 \\
\hline $46 \mathrm{R}$ & CPM/FOSF & $256 / 512$ & $\leq 1 / 16$ & 0.035 \\
\hline $46 \mathrm{R}$ & CPM/RIF & $>512 / 32$ & $32 / \leq 4$ & 0.156 \\
\hline $46 \mathrm{R}$ & TOB/FOSF & $512 />256$ & $128 / 32$ & 0.313 \\
\hline $46 \mathrm{R}$ & TOB/RIF & $512 / 32$ & $16 / \leq 4$ & 0.156 \\
\hline $72 \mathrm{R}$ & IPM/GEN & $512 / 128$ & $2 / 8$ & 0.066 \\
\hline $72 \mathrm{R}$ & IPM/FOSF & $>512 / 128$ & $256 / 32$ & 0.500 \\
\hline $72 \mathrm{R}$ & IPM/RIF & $512 / 32$ & $128 / \leq 4$ & 0.375 \\
\hline $72 \mathrm{R}$ & PIP-T/TOB & $>512 / 256$ & $64 / 32$ & 0.188 \\
\hline $72 \mathrm{R}$ & PIP-T/FOSF & $>512 / 256$ & $4 / 64$ & 0.254 \\
\hline $72 \mathrm{R}$ & CEFTA/TOB & $>512 />256$ & $128 / 32$ & 0.188 \\
\hline $72 \mathrm{R}$ & CEFTA/FOSF & $>256 / 512$ & $128 / 128$ & 0.500 \\
\hline $72 \mathrm{R}$ & CPM/TOB & $512 / 256$ & $64 / 32$ & 0.250 \\
\hline $72 \mathrm{R}$ & $\mathrm{CIP} / \mathrm{TOB}$ & $16 / 256$ & $4 / 16$ & 0.313 \\
\hline $72 \mathrm{R}$ & $\mathrm{CIP} / \mathrm{FOSF}$ & $16 / 256$ & $2 / 16$ & 0.188 \\
\hline $72 \mathrm{R}$ & TOB/FOSF & $>512 / 256$ & $16 / 32$ & 0.141 \\
\hline $72 \mathrm{R}$ & TOB/RIF & $512 / 32$ & $8 / \leq 4$ & 0.141 \\
\hline
\end{tabular}

IPM: imipenem; PIP-T: piperacillin-tazobactam; CEFTA: ceftazidime; TOB: tobramycin; GEN: gentamicin; CIP: ciprofloxacin; FOSF: fosfomycin; RIF: rifampin.

\section{DISCUSSION}

This study revealed that various antimicrobial combinations could act synergistically in vitro against multidrug-resistant Brazilian P. aeruginosa isolates. Polymyxins and fosfomycin are old antibiotics that have been retrieved in an attempt to control 
TABLE 3 - In vitro synergistic combinations of antimicrobials against four strains of Pseudomonas aeruginosa.

\begin{tabular}{|c|c|c|c|c|c|c|c|c|c|}
\hline \multicolumn{10}{|c|}{ Number of strains with synergy } \\
\hline \multirow{3}{*}{$\frac{\text { Drug B }}{\text { GEN }}$} & \multicolumn{7}{|c|}{ Drug A } & \multirow{2}{*}{\multicolumn{2}{|c|}{$\begin{array}{c}\text { Synergistic/all } \\
\text { tests n }(\%)\end{array}$}} \\
\hline & IPM & PIP-T & CEFTA & CPM & CIP & GEN & TOB & & \\
\hline & 1 & 2 & 1 & 1 & 0 & & & $5 / 20$ & 25.0 \\
\hline TOB & 3 & 4 & 3 & 2 & 1 & & & $13 / 20$ & 65.0 \\
\hline FOSF & 4 & 4 & 3 & 1 & 2 & 0 & 2 & $16 / 28$ & 57.0 \\
\hline RIF & 3 & 1 & 2 & 1 & 0 & 0 & 3 & $10 / 28$ & 36.0 \\
\hline Synergistic/all tests n (\%) & $11 / 16(69.0)$ & $11 / 16(69.0)$ & $9 / 16(56.0)$ & $5 / 16(31.0)$ & $3 / 16(19.0)$ & $0 / 8(0.0)$ & $5 / 8(63.0)$ & $44 / 96$ & 46.0 \\
\hline
\end{tabular}

IPM: imipenem; PIP-T: piperacillin/tazobactam; CEFTA: ceftazidime; CPM: cefepime; GEN: gentamicin; TOB: tobramycin; FOSF: fosfomycin; RIF: rifampin; CIP: ciprofloxacin. $\mathrm{p}<0.05$

nosocomial infections caused by this microorganism ${ }^{10-11-12}$, but isolates with high MICs for these drugs have already been detected ${ }^{1-13-14}$. The increasing number of infections related to multidrug-resistant $P$. aeruginosa in different areas of the world ${ }^{4-15-16}$ has stimulated the investigation of the synergy of antimicrobial combinations. The checkerboard method is widely employed for this purpose and was modified in this study to measure the growth in microplates as absorbance units. The results obtained with this instrumental technique show a good correlation with the visual reading and provide a better, more objective definition of the intermediate zone of growth and therefore, more precise MIC determinations for combined drugs.

The overall rate of synergy observed in this study was $46 \%$. The combinations of imipenem, piperacillin-tazobactam and ceftazidime with a second drug often resulted in synergy. Combinations of these antibiotics with tobramycin exhibited synergy in $83 \%$ of the tests performed with the four P. aeruginosa isolates. In another study assessing multidrug-resistant P. aeruginosa, ceftazidime plus tobramycin and piperacillintazobactam plus tobramycin combinations were evaluated, and synergy ratios of $67 \%$ and $50 \%$, respectively, were observed ${ }^{17}$. With respect to fosfomycin, synergistic interactions with other antibacterial drugs were verified in $57 \%$ of the tests, a rate similar to that reported previously for multidrug-resistant P. aeruginosa ${ }^{18}$. Fosfomycin enhances the active transport of tobramycin in P.aeruginosa ${ }^{19}$; in vitro synergic actions were also demonstrated for polymyxin $\mathrm{E}^{10}$, imipenem ${ }^{20}$, ceftazidime ${ }^{20}$ and ciprofloxacin ${ }^{21}$. Previous studies showed that rifampin had in vitro synergism with other antimicrobials ${ }^{22-25}$, whereas in this investigation, synergism was more frequently demonstrated with imipenem and aminoglycosides.

The clinical application of the in vitro synergy results must be considered with caution in view of the variable susceptibilities of $P$. aeruginosa isolates to combined drugs and the pharmacokinetic characteristics of the antimicrobials. As observed in other studies ${ }^{26,27}$, the rate of synergy of antibacterial combinations varies according to isolate and is not strictly associated with susceptibility or resistance to imipenem. Comparison of the two multidrug-resistant $P$. aeruginosa isolates revealed more frequent and significant drug MIC reductions for the $46 \mathrm{R}$ isolate than for the $72 \mathrm{R}$ isolate. Thus, it is advisable to test each multidrug-resistant isolate with the different drugs in combination ${ }^{17,28}$.

Among the synergy results, only a few antibacterial combinations have led to sufficient MIC reductions that reach the breakpoint and the usual plasma level of the drugs, which is essential if a synergistic action in a clinical setting is going to take place ${ }^{5,28}$. For the $72 \mathrm{R}$ isolate, the only antibacterial combinations that would likely be synergic in vivo are imipenem plus gentamicin, piperacillin-tazobactam plus fosfomycin, ciprofloxacin plus fosfomycin, tobramycin plus fosfomycin and tobramycin plus rifampin.

In conclusion, antimicrobial synergy was observed against clinical isolates of imipenem-susceptible or imipenem-resistant $P$. aeruginosa. Some drug combinations resulted in sufficient MIC reductions, which suggests that these combinations may be of clinical use for infections of multidrug-resistant $P$. aeruginosa as an alternative to antibiotic therapy with polymyxins.

\section{CONFLICT OF INTEREST}

The authors declare that there is no conflict of interest.

\section{FINANCIAL SUPPORT}

This research was supported by Fundação de Apoio ao Ensino, Pesquisa e Assistência do Hospital das Clínicas, Faculdade de Medicina de Ribeirão Preto, Universidade de São Paulo.

\section{REFERENCES}

1. Ham VH, Chang KT, Abdelrouf K, Brioso CG, Ameka M, McCaskey LA, et al. Prevalence, resistance mechanisms, and susceptibility of multidrugresistant bloodstream isolates of Pseudomonas aeruginosa. Antimicrob Chemother 2010; 54:1160-1164.

2. Marra AR, Camargo LF, Pignatari AC, Sukienni KT, Bahar PR, Medeiros EA, et al. (Brazilian SCOPE Study Group). Nosocomial blood stream infections in Brazilian hospitals: analysis of 2,563 cases from a prospective nationwide surveillance study. J Clin Microbiol 2011; 49:1866-1871.

3. Jácome PRLA, Alves LR, Cabral AB, Lopes ACS, Maciel MAV. Phenotypic and molecular characterization of antimicrobial resistance 
and virulence factors in Pseudomonas aeruginosa clinical isolates from Recife, State of Pernambuco, Brazil. Rev Soc Bras Med Tropical 2012; 45:707-712.

4. Tamma PD, Cosgrove SE, Maragakis LL. Combination therapy for treatment of infections with gram-negative bacteria. Clin Microbiol Rev 2012; 25:450-470.

5. Drusano GL, Bonomo RA, Bahnink N, Bulitta JB, Vanscoy B, DeFiglio H, et al. Resistance emergence mechanism and mechanism of resistance suppression by tobramycin for cefepime for Pseudomonas aeruginosa. Antimicrob Ag Chemother 2012; 56:231-242.

6. Clinical and Laboratory Standards Institute (CLSI). Methods for dilution antimicrobial susceptibility tests for bacteria that grow aerobically. Eighth edition: Aproved Standard M07-A8. Wayne, PA, USA: CLSI; 2009.

7. Clinical and Laboratory Standards Institute (CLSI). Performance standards for antimicrobial susceptibility testing.Twentieth Informational Supplement M100-S20. Wayne, PA, USA: CLSI; 2010.

8. European Committee on Antimicrobial Susceptibility Testing (EUCAST). Breakpoints tables for interpretation of MICs and zone diameters [Software]. EUCAST 2013. [Version 3.0]. Available from: http://www. eucast.org/clinical_breakpoints/.

9. Foweraker JE, Laughton CR, Brown DF, Bilton D. Comparison of the methods to test antibiotic combinations against heterogeneous populations of multiresistant Pseudomonas aeruginosa from patients with acute infective exacerbations in cystic fibrosis. Antimicrob Ag Chemother 2009; 53:4809-4815.

10. Maviglia R, Nestorini R, Pennisi M. Role of old antibiotics in multidrug resistant bacterial infections. Curr Drug Targets 2009; 10:895-905.

11. Furtado GH, D'Azevedo PA, Santos AF, Gales AC, Pignatari AC, Medeiros EA. Intravenous polymyxin for the treatment of nosocomial pneumonia caused by multidrug-resistant Pseudomonas aeruginosa. Int J Antimicrob Agents 2007; 30:315-319.

12. Samonis G, Maraki S, Rafailidis PI, Kaspaskelis A, Kastoris AC, Falagas ME. Antimicrobial susceptibility of Gram-negative non urinary bacteria to fosfomycin and other antimicrobials. Future Microbiol 2010; 5: 961-970.

13. De Groote VN, Faunart M, Kint CL, Verstaetein N, Jans A, Cornelis P, et al. Pseudomonas aeruginosa fosfomycin resistance mechanisms affect noninherited fluoroquinolone tolerance. J Med Microbiol 2011; 60: 329-336.

14. Rodriguez-Rojas A, Maciá MD, Couce A, Goméz C, Castañeda-Garcia A, Oliver A, et al. Assessing the emergence of resistance: the absence of biological cost in vivo may compromise fosfomycin treatments for P. aeruginosa infections. PloS One 2010; 5:e10193.

15. Gales AC, Menezes LC, Silbert S, Sader HS. Dissemination in distint Brazilian regions of an epidemic carbapenem-resistant Pseudomonas aeruginosa producing SPM metallo-beta-lactamase. J Infect Chemother 2003; 52:699-702.
16. Timurkaynak F, Can F, Azap OK, Demirbilek M, Arslan H, Karaman SO. In vitro activities of non-traditional antimicrobials alone or in combination against multidrug-resistant strain Pseudomonas aeruginosa and Acinetobacter baumannii isolated from intensive care units. Int $\mathrm{J}$ Antimicrob Agents 2006; 27:224-228.

17. Dundar D, Otkum M. In vitro efficacy of synergistic antibiotic combinations in multidrug resistant Pseudomonas aeruginosa strains. Yonsei Med J 2010; 51:111-116.

18. Falagas MF, Kastoris AC, Karageouropoulos DE, Rafailidis P. Fosfomycin for the treatment of infections caused by multidrug-resistant nonfermenting gram-negative bacilli: a systematic review of microbiological, animal and clinical studies. Int J Antimicrob Agents 2009; 34:111-120.

19. MacLeod DL, Velayudhan J, Kenney TF, Therrien JH, Sutherland JL, Barker LM, et al. Fosfomycin enhances the active transport of tobramycin in Pseudomonas aeruginosa. Antimicrob Ag Chemother 2012; 56:1529-1538.

20. Pruekpreasert $\mathrm{P}$, Tumiapanit W. In vitro activity of fosfomycin-gentamicin, fosfomycin-ceftazidime, fosfomycin-imipenem and ceftazidime-gentamicin combinations against ceftazidime-resistant Pseudomonas aeruginosa. Southeast Asian Trop Med Publ Health 2005; 36:1239-1242.

21. Yamada S, Hyo Y, Ohuori M. Role of ciprofloxacin in its synergistic effect with fosfomycin on drug-resistant strains of Pseudomonas aeruginosa. Chemotherapy 2007; 53:202-209.

22. Chin NX, Neu HC. Synergy of impenem -a novel carbapenem, and rifampin and ciprofloxacin against Pseudomonas aeruginosa, Serratia marcescens and Enterobacter species. Chemotherapy 1987; 33:183-188.

23. Valdes JM, Baltch AL, Smith RP, Hammer MC, Ritz WJ. The effect of rifampicin on the in vitro activity of cefpirome or ceftazidime in combination with aminoglycosides against Pseudomonas aeruginosa. J Antimicrob Chemother 1990; 25:575-584.

24. Xiong YQ, Caillon J, Drugcon H, Potel G, Baron D. The effect of rifampicin on adaptative resistance of Pseudomonas aeruginosa to aminoglycosides. J Antimicrob Chemother 1996; 37:993-998.

25. Giamarellos-Bourbouilis EJ, Sambatakon H, Galani I, Giamarellou H. In vitro interaction of colistin and rifampin on multidrug resistant Pseudomonas aeruginosa. J Chemother 2003; 15:235-238.

26. Weiss K, Lapointe JR. Routine susceptibility testing of four antibiotic combinations for improvement of laboratory guide to therapy of cystic fibrosis infections caused by Pseudomonas aeruginosa. Antimicrob Ag Chemother 1995; 39:2411-2414.

27. Kanellakopoulou K, Sarafis P, Galani I, Giamarellou H, GiamarellosBourboulis EJ. In vitro synergism of beta-lactams with ciprofloxacin and moxifloxacin against genetically distint multidrug-resistant isolates of Pseudomonas aeruginosa. Int J Antimicrob Agents 2008; 32:33-39.

28. Yuan Z, Ledesma KR, Singh R, Prince RA, Tam VH. Quantitative assestment of combination antimicrobial therapy against multidrugresistant bacteria in a murine pneumonia model. J Infect Dis 2010; 201:889-897. 